\title{
Integrated optical waveguides and inertial focussing microfluidics in silica for microflow cytometry applications
}

Jonathan T. Butement, ${ }^{1 *}$ Hamish C. Hunt, David J. Rowe, ${ }^{1}$ Neil P. Sessions, ${ }^{1}$ Owain Clark, ${ }^{2}$ Ping Hua, ${ }^{1}$ G. Senthil Murugan, ${ }^{1}$ John E. Chad, ${ }^{3,4}$ and James S. Wilkinson ${ }^{1,4}$

${ }^{1}$ Optoelectronics Research Centre, University of Southampton, Southampton SO17 1BJ, UK

${ }^{2}$ Electronics and Computer Science, University of Southampton, Southampton SO17 1BJ, UK

${ }^{3}$ Centre for Biological Sciences, University of Southampton, Southampton SO17 1BJ, UK

${ }^{4}$ Institute for Life Sciences, University of Southampton, Southampton SO17 1BJ, UK

*Corresponding author: jb3006@soton.ac.uk

Received Month X, XXXX; revised Month X, XXXX; accepted

Month X, XXXX; posted Month X, XXXX (Doc. ID XXXXX);

A key challenge in the development of a microflow cytometry platform is the integration of the optical components with the fluidics as this requires compatible micro-optical and microfluidic technologies. In this work a microflow cytometry platform is presented comprising monolithically integrated waveguides and deep microfluidics in a rugged silica chip. Integrated waveguides are used to deliver excitation light to an etched microfluidic channel and also collect transmitted light. The fluidics are designed to employ inertial focussing, a particle positioning technique, to reduce signal variation by bringing the flowing particles onto the same plane as the excitation light beam. A fabrication process is described which exploits microelectronics mass production techniques including: sputtering, ICP etching and PECVD. Example devices were fabricated and the effectiveness of inertial focussing of $5.6 \mu \mathrm{m}$ fluorescent beads was studied showing lateral and vertical confinement of flowing beads within the microfluidic channel. The fluorescence signals from flowing calibration beads were quantified demonstrating a CV of $26 \%$. Finally the potential of this type of device for measuring the variation in optical transmission from input to output waveguide as beads flowed through the beam was evaluated.

\section{KEYWORDS}

Integrated optics

Microflow cytometry

Inertial focussing

Bead-based immunoassay 


\section{INTRODUCTION}

Flow cytometry is an important tool for medicine and biology, with applications from clinical diagnosis to investigations of fundamental cell biology. However, traditional flow cytometers are expensive, bulky and complex to operate as they are an assembly of macroscale electronic, optical and fluidic components. Microflow cytometers, which combine miniaturised fluidic and detection systems on a chip would offer advantages over traditional devices, being compact, cheap and massproducible and would offer the user ease of operation and portability [1].

Both electrical and optical analysis techniques are used in flow cytometry to measure the size, morphology and biological function of cells or particles. The electrical impedance of a particle can be used to sensitively determine particle size down to submicron diameters [2] and impedance spectroscopy can be used for label free cell differentiation to a limited extent [3]. Optical analysis can also be used to measure the size and morphology from the angular scatter of particles and offers more information on phenotype than impedance sensing as a range of fluorescently tagged antibodies with different emission peaks can be used to discriminate between cell types. While impedance based detection can be readily integrated into planar chip configurations using planar electrodes [4] integration strategies for optofluidics have not been fully developed [5]. A popular approach is to insert optical fibres into microchannels aligned with the microfluidics [6-8]. While this exploits the low cost and well characterised performance of commercial fibres, the insertion of the fibres is a manual process which is not suited to mass production.

An alternative approach, undertaken in this work, is to monolithically integrate waveguides with microfluidics by fabricating both on the same substrate using mass production techniques [9]. This is particularly advantageous when photolithographically based fabrication processes are used as the optical and fluidic structures are permanently aligned with submicron precision. Much of the work on monolithic integration has used polymer-based material systems such as negative photoresist $[10,11]$ or embossed thermoplastics [12] to define the waveguides and fluidics. Whilst polymer based system are often cheaper and quicker to fabricate, glass based integration systems are appealing as they can offer superior optical performance and mechanical stability making them attractive as a cost effective replacement to high precision laboratory instruments. The material stability offers the potential to include advanced integrated optical components which are sensitive to dimensional changes, such as kinoform lenses [13], multimode interference devices [14] and spectrometers [15] for light focussing, collection and analysis.

Glass based optofluidic and cytometry platforms which exploit the mass production processes used by the microelectronics industry have been demonstrated $[16,17]$. However limited attention has been paid to a suitable microfluidic particle focussing strategy which can be easily fabricated in these materials. One particle focussing strategy which shows promise for integration in glass is inertial particle focussing $[18,19]$. In this technique, shown in Figure 1, microparticles flowing in a straight, rectangular cross-section channel at a sufficiently high velocity are subjected to lateral lift forces which cause them to migrate across the flow streamlines to equilibrium positions. The two main lift forces, shown in Figure 1(a), are the shear gradient lift force and the wall effect lift force. The shear gradient lift force acts to move the particle away from the central axis of the channel. This force arises from the parabolic fluid velocity profile in the microfluidic channel. The velocity of the particle lags the fluid velocity resulting in a differential relative fluid velocity across the particle diameter creating a net force down the fluid velocity gradient towards the channel wall. The wall effect lift force acts to move the particle toward the central axis of the channel. The particle lagging behind the flow causes the fluid streamlines to be directed around the particle. The fluid passing over the particle on the side facing the centre of the channel is accelerated around the particle resulting in a lower pressure compared to the fluid passing by the more direct route between the particle and the 
wall producing a net force acting away from the wall [2]. Lift forces become significant when the particle Reynolds number, which describes the ratio of inertial to viscous forces on the particle length scale, is of order 1 causing particle migration to the equilibrium positions where the forces are balanced [20]. In a rectangular cross section microchannel, shown in Figure 1(b), there are two equilibrium positions centred on the long faces of the channel cross-section.

Inertial focussing has advantages over other particle positioning techniques such as hydrodynamic focussing [21] and electrodynamic focussing [22] as the channels are simpler to fabricate and control of only one fluidic input is required. Another useful aspect of inertial focussing is that particles at an optimum concentration become evenly spaced in the direction of flow and are positioned alternately on each side of the channel, thereby preventing simultaneous signals from overlapping particles [23]. The use of inertial focussing in microflow cytometry is growing, with examples using free space optics [24,25] and also with polymer based integrated optics [12]. Currently there have been no demonstrations of inertial focussing combined with waveguides in glass based systems.

a)

b)
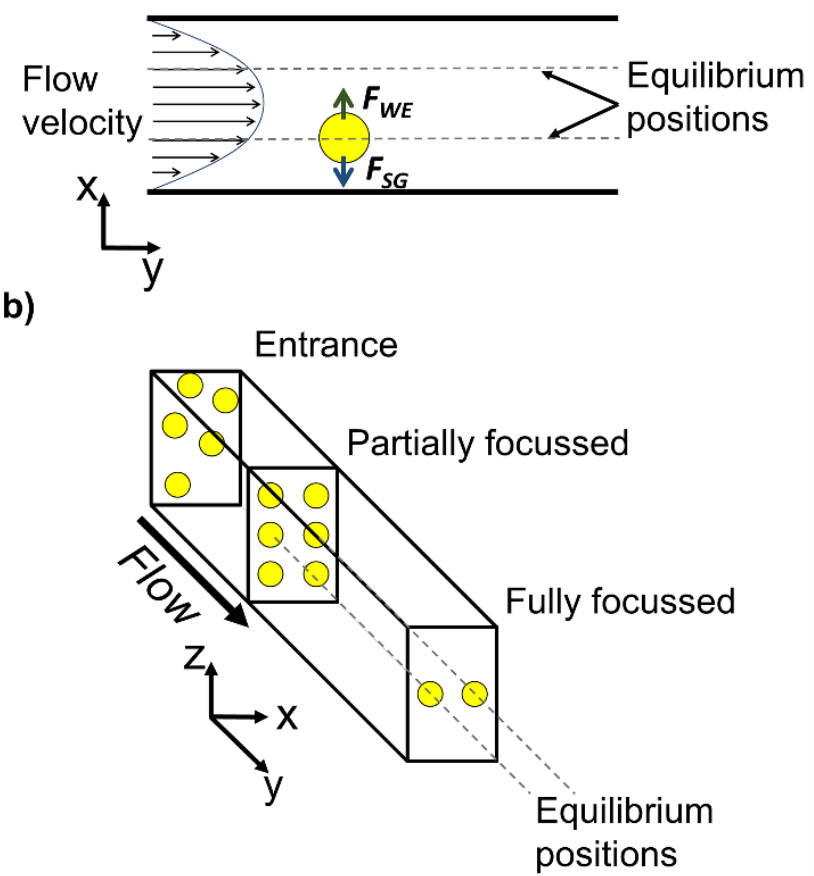

Figure 1. (a) The shear gradient lift force $\left(F_{S G}\right)$ and wall effect lift force $\left(F_{W E}\right)$ act on a flowing microparticle to produce inertial focussing at an equilibrium position. (b) A rectangular cross-section microfluidic channel produces two equilibrium positions centred on the long faces of the channel cross section.

In this paper a silica based microflow cytometry platform is presented which integrates waveguides with deep microfluidic channels suitable for inertial particle focussing. To demonstrate a relevant application, an example device is fabricated and used for fluorescence and transmission based detection of flowing polystyrene microspheres (beads) commonly used in bead-based immunoassays [26]. In the future it is envisaged that more specialised designs could be used for the analysis of cells and extracellular vesicles $[27,28]$, building on previous work which has developed integrated lenses in the same material system [14]. The optical beam shape and inertial focussing behaviour of beads in the microfluidic channel are characterised. Finally the transmission-based detection of flowing beads is demonstrated which has potential uses for the measurement of particle count rate, bead size and flow velocity and opens the way to future forward-scatter and side-scatter designs. 


\subsection{Device design and operation}

The example flow cytometry device used in this paper consists of a silica chip with embedded strip waveguides bisected orthogonally by a deep microfluidic channel, as shown in Figure 2(a). To allow efficient coupling to commercial single-mode optical fibres, the refractive index of the waveguide core material was chosen to be similar to that of the fibre core $(\Delta n \approx 0.7 \%)$ and the waveguide height was chosen to be $2 \mu \mathrm{m}$ to offer good matching to the fibre mode [14]. The waveguides are grouped into arrays along the length of the fluidic channel. Each array has waveguides varying in width from $1.8 \mu \mathrm{m}$ to $9.2 \mu \mathrm{m}$. This design allows for a range of excitation beam widths to be launched across the fluidic channel and single mode to multimode operation at wavelengths of 532 $\mathrm{nm}$ and $635 \mathrm{~nm}$. These wavelengths are appropriate for fluorescence excitation in a typical beadbased immunoassay using commercial bead systems. The microfluidic channel was designed to inertially focus beads of 5-6 $\mu \mathrm{m}$ diameter within a maximum downstream length of $30 \mathrm{~mm}$ whilst remaining practical to fabricate. The channel is $30 \mathrm{~mm}$ long and is of a rectangular cross-section 20 $\mu \mathrm{m}$ wide and $30 \mu \mathrm{m}$ deep, as shown in Figure 2(b). The waveguides are positioned so that their end facets are halfway up the sidewalls of the microfluidic channel with the centre of the waveguide core $15 \mu \mathrm{m}$ above the floor of the channel. Inertial focussing acts to position the flowing beads at two equilibrium positions which are on the plane of the optical axis. This vertical and horizontal confinement of flowing beads will reduce variation in excitation power encountered by each bead and thus reduce fluorescence signal variation. Fluorescence collection and measurement is achieved with a microscope and CCD camera positioned above the chip. This mode of collection was required to allow imaging of the inertial focussing behaviour of beads with simultaneous quantification of fluorescence. Future work will develop a compact method, higher speed method for fluorescence collection. Transmission based detection of flowing beads is enabled by the axially aligned output waveguide which collects light from the excitation beam. Beads flowing through the beam deflect and attenuate the excitation beam and cause modulation in the power collected by the output waveguide. This has potential to allow determination of the total count rate of beads, recognition of double signals and synchronisation of fluorescence date collection for noise reduction. For fully inertially focussed beads flowing on the optical axis the reduction in transmitted power will be directly related to bead diameter, allowing this signal to be used to identify bead or cell size. 


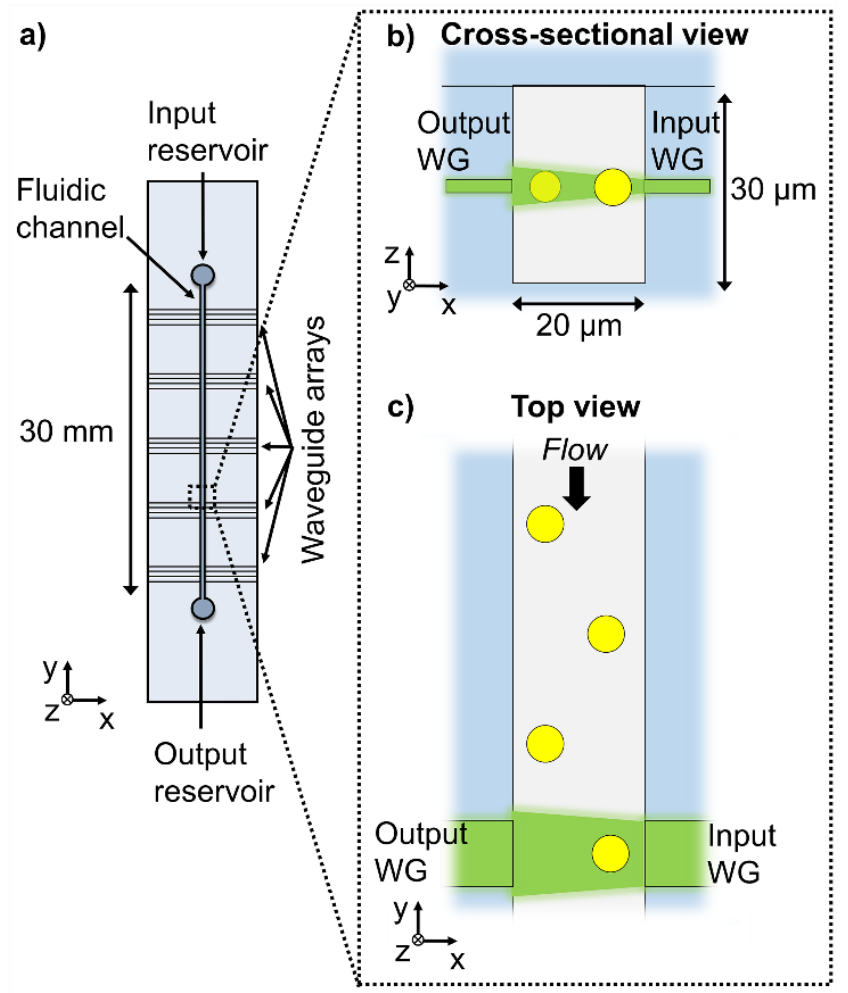

Figure 2. (a) Plan view schematic of the microflow cytometer device; (b) Channel cross-sectional view of inertiallyfocussed beads and waveguide (WG) positions; (c) inertially focussed beads from top (plan) view.

\section{METHODS}

\subsection{Fabrication}

After preliminary process optimisation, the following protocol was adopted to fabricate the flow cytometry devices. Silica substrates were cleaned and a $2 \mu \mathrm{m}$ thick germania-doped silica waveguide layer (25:75 wt\% $\mathrm{GeO}_{2}: \mathrm{SiO}_{2}$ ) was deposited by RF magnetron sputtering (Plasmalab 300, OIPT, UK). The deposition parameters were: chamber pressure $10 \mathrm{mTorr}$, magnetron power $300 \mathrm{~W}$, gas flow rate $\mathrm{O}_{2} / \mathrm{Ar}=5 / 20 \mathrm{sccm}$ and chamber temperature $200^{\circ} \mathrm{C}$. The waveguide layer was then annealed at $950^{\circ} \mathrm{C}$ for 6 hours in a tube furnace with a ramp up/down rate of $5{ }^{\circ} \mathrm{C} / \mathrm{min}$ under an oxygen atmosphere, to reduce oxygen deficiency and thereby lower optical losses. Lift-off photolithography and e-beam evaporation were used to define a $300 \mathrm{~nm}$ thick chromium hard mask on the waveguide film. A patterned photoresist bilayer of $300 \mathrm{~nm}$ thick LOR-3B (Microchem Corp, USA) topped with 1.3 $\mu \mathrm{m}$ thick S1813 (Dow Electronic Materials, USA) was used for the lift-off process. Strip waveguide structures were created by inductively coupled plasma (ICP) etching through the entire waveguide film to a depth of $2.4 \mu \mathrm{m}$ (to ensure full etching) using a Plasmalab 100 ICP380 system (OIPT, UK). The etch parameters were: chamber pressure $10 \mathrm{mTorr}$, ICP power $2500 \mathrm{~W}$, RF bias power $70 \mathrm{~W}$, gas flow rates $\mathrm{C}_{4} \mathrm{~F}_{8} / \mathrm{O}_{2}=40 / 15 \mathrm{sccm}$ and table temperature $45^{\circ} \mathrm{C}$. The resulting strip waveguides were clad with $18 \mu \mathrm{m}$ of $\mathrm{SiO}_{2}$ deposited by plasma enhanced chemical vapour deposition (PECVD) on a Plasmalab 100 LDS-PECVD system (Oxford instruments, UK). The deposition parameters were: chamber pressure $1000 \mathrm{mTorr}$, RF power $20 \mathrm{~W}$, table temperature of $350{ }^{\circ} \mathrm{C}$ and gas flow $\mathrm{N}_{2} \mathrm{O} / \mathrm{SiH}_{4}=$ $2000 / 4 \mathrm{sccm}$. To prevent stress-induced cracking, the cladding was deposited in steps of thickness of up to $5 \mu \mathrm{m}$ at a time followed by annealing employing the same parameters as for the waveguide core. The chip was planarised to reduce the height of the cladding ridges by chemical-mechanical polishing on an LP-50 polishing machine (Logitech, UK), removing $3 \mu \mathrm{m}$ of cladding thickness. A 1.3 $\mu \mathrm{m}$ thick chromium hard mask for etching of the microfluidic channels was defined using lift-off lithography with e-beam deposition. Here, a patterned photoresist bilayer consisting of $2.8 \mu \mathrm{m}$ thick 
LOR-30B topped with $1.3 \mu \mathrm{m}$ thick S1813 was used for the lift-off process. To prevent stress induced peeling of the chromium, the film was deposited in steps of no more than $200 \mathrm{~nm}$ thickness at a time followed by a 10 minute break [29]. The chromium deposition rate was $0.65 \mu \mathrm{m} / \mathrm{hr}$. The channels were then ICP etched to the required depth of $30 \mu \mathrm{m}$, using the same process as that used to etch the waveguide films and the chromium was removed by wet etching. All feature heights were measured either using a surface profiler ( $p-16$, KLA Tencor, USA) for low aspect ratio features or using an optical profiler (Zescope, Zemetrics,USA). To measure film deposition rates, the surface profiler was used to measure the deposited film height with reference to an area of the substrate masked during deposition. The refractive indices of the substrate and films were measured at $\lambda=$ $633 \mathrm{~nm}$ using a prism coupler (2010/M, Metricon Corp., USA). Individual chips were diced using a wafer-dicing saw and the end facets of the waveguides lapped and polished. To seal the microfluidic channel, glass coverslips of $170 \mu \mathrm{m}$ thickness with predrilled access holes were spin coated with a 2 $\mu \mathrm{m}$ thick layer of SU8-2002 photoresist (Microchem, USA) and soft baked at $72{ }^{\circ} \mathrm{C}$ on a hot plate. During the soft bake the top surface of the chip was placed into contact with photoresist and pressure was applied with weights for 20 minutes. Once full contact was achieved the chip was removed from the hotplate and the photoresist was UV cured.

\subsection{Optical characterisation}

The apparatus shown in Figure 3 was used for optical characterisation of the device and for quantifying fluorescence from flowing beads. The apparatus consisted of a microscope with a 20x, 0.46 NA microscope objective lens focussed on the region of the microfluidic channel illuminated by the waveguide, a $542 \mathrm{~nm}$ long pass filter to cut out excitation light and CCD camera (ORCA-R2, Hamamatsu, Japan). The device chip was mounted with the non-microstructured side of the substrate facing the microscope objective. Light from a $20 \mathrm{~mW}$ fibre coupled diode pumped solidstate, 532nm laser (OZ-2000-532, OZ Optics, Canada) was coupled into the input waveguide via a butt-coupled single mode fibre with polarisation control. Light leaving the output waveguide was collected by a microscope objective lens and focussed through a TE oriented polariser and then through a pinhole, to minimise stray light, onto an optical power meter. TE input polarisation was selected by manipulating the polarisation controller until the output power was maximum. Initial measurements to determine the maximum waveguide width for monomode operation were carried out using the same apparatus with the CCD camera placed after OBJ 2 and observing the near-field intensity distribution at the waveguide output while translating the input fibre across the input facet. The optical insertion loss incurred by the microfluidic channel was measured by imaging the autofluorescence from the input and output waveguides on the CCD camera and using image analysis in MATLAB to determine the relative intensity of waveguide autofluorescence immediately before and after the microfluidic channel. Imaging of the beam crossing the microfluidic channel was achieved by pumping a solution of the fluorescent dye R-phycoerythrin $(0.4 \mu \mathrm{M}$ in distilled water with $0.2 \% \mathrm{v} / \mathrm{v}$ Tween20 surfactant) through the channel and imaging from above with the microscope and CCD camera. 


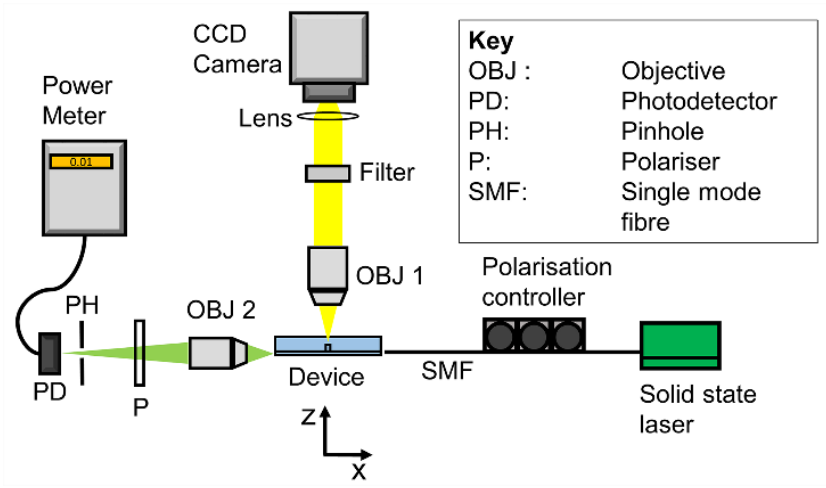

Figure 3. Schematic diagram of the apparatus used for optical characterisation of the device and to measure fluorescence from flowing beads

\subsection{Measurement of bead fluorescence}

Prior to the introduction of a bead suspension, water was pumped through the microfluidic channel at a rate of $0.2 \mu \mathrm{L} / \mathrm{s}$ for 10 minutes to remove any air bubbles. Bead suspensions were pumped to the chip using a syringe pump (Legato 110, KDS, USA.) through a $10 \mu \mathrm{m}$ pore size filter to remove clusters and then through tubing coupled to the chip input reservoir. A video of the channel area illuminated by the waveguide was recorded with an exposure time of $0.0125 \mathrm{~s}$, at a rate of 79.5 frames per second. There was an interval of $78 \mu$ s between frames where no light was acquired by the camera whilst the pixels were read out, which represents $<1 \%$ of the exposure time. The time taken for a bead to pass through the excitation beam ( 30 $\mu \mathrm{s})$ is much shorter than the frame exposure time, therefore producing a "streak" image. The sum of the energy recorded in each pixel of the "streak" gives the total fluorescence energy emitted by the bead as it passes through the illumination beam, and this was calculated for each frame by post-processing in MATLAB. A rectangular box was defined around the area in the image illuminated by the waveguide and all the pixel values within this box were summed, for each frame, giving the total fluorescence energy collected in 16-bit digital units. The contribution to the signal from chip autofluorescence and intrinsic background in the camera was eliminated by subtracting the summed pixel values of the region of interest when only buffer solution was flowing. This background corrected, fluorescence energy value in 16-bit digital units was converted to joules according to the camera specifications [30]. No digital filters were applied to the raw fluorescence energy signal. Fluorescence energy signals which exceeded a threshold of ten standard deviations above the mean fluorescence energy signal when only buffer was flowing were deemed to be bead signals.

\subsection{Inertial focussing characterisation}

The extent to which inertial focussing achieved confinement of flowing beads was characterised using a waveguide located $22 \mathrm{~mm}$ downstream from the microfluidic channel entrance, by which distance inertial focussing is expected to be established [18]. A suspension of $5.6 \mu \mathrm{m}$ diameter fluorescent beads (Inspeck Orange, ThermoFisher, USA) in distilled water with $0.2 \% \mathrm{v} / \mathrm{v}$ Tween20 surfactant was prepared at a concentration of 10 beads/ $\mu \mathrm{L}$. The suspension was flowed through the device at $0.2 \mu \mathrm{L} / \mathrm{s}$ and a video was acquired of the beads passing the illuminated channel region. The bead concentration was selected to ensure the occurrence of coincident signals remained below $2 \%$ of the total number of signal peaks. Inertial focussing parallel to the short face of the microfluidic channel cross-section ( $X$ axis) was examined using pixel by pixel summing of all the video frames containing a bead into a single image to give the lateral distribution of beads passing through the beam. Inertial focussing parallel to the long face of the microfluidic channel cross-section (z-axis) was assessed by comparing the count rate of beads detected to the maximum possible count rate determined by the bead concentration and flow rate. The input waveguide used was $9.2 \mu \mathrm{m}$ wide, to provide the longest illumination duration and maximise the amount of fluorescent energy collected. 


\subsection{Measurement of bead transmission signal}

To measure the effect of beads flowing through the beam on the optical transmission across the microfluidic channel, the output waveguide was butt-coupled to a $60 \mu \mathrm{m}$ diameter core multimode fibre connected to an avalanche photodetector (APD) (APD120A2/M, Thorlabs, USA) with an receiver bandwidth of $50 \mathrm{MHz}$. A bead suspension (Inspeck Orange, ThermoFisher, USA) at 10 beads/ $\mu$ l concentration was prepared and flowed through the device whilst the waveguide output power was recorded. Data was recorded for flow rates of $0.2 \mu \mathrm{L} / \mathrm{s}$, when beads are inertially focussed and at $0.05 \mu \mathrm{L} / \mathrm{s}$ when beads are not inertially focused and the bead positioning is random, for the purpose of comparison. The power transmitted across the fluidic channel and collected by the output waveguide was acquired multiple times on a digital oscilloscope recording at a rate of 5 $\mathrm{MS} / \mathrm{s}$ with a storage capacity of $10^{5}$ data points per manual acquisition. No digital filters were applied to the transmission signals.

\section{$3 \quad$ RESULTS AND DISCUSSION}

\subsection{Fabrication}

The material deposition rates were found to be $0.26 \mu \mathrm{m} / \mathrm{h}$ for the sputtered germania:silica core and $1.92 \mu \mathrm{m} / \mathrm{h}$ for the PECVD silica cladding. The cladding refractive index was controlled using the deposition gas flow ratios, and increasing the $\mathrm{N}_{2} \mathrm{O} / \mathrm{SiH}_{4}$ flow ratio led to decreasing film refractive index. This trend is in agreement with $\mathrm{Kim}$ et al [31]. The refractive indices of the optical layers, measured by prism coupling (Metricon 2010/M, Metricon Corp, USA), were 1.4570 \pm 0.0001 , $1.4690 \pm 0.0007$ and $1.4571 \pm 0.0001$ for substrate, core and cladding respectively at $\lambda=633 \mathrm{~nm}$. $A$ $\mathrm{N}_{2} \mathrm{O} / \mathrm{SiH}_{4}$ flow ratio of 500:1 was found to produce annealed silica films with refractive indices within $\pm 0.1 \%$ of the bulk silica. The ICP etch process yielded etch rates for bulk silica, germania:silica, PECVD silica and chromium of $0.22,0.39,0.26$ and $0.005 \mu \mathrm{m} / \mathrm{min}$ respectively. Figure 4 shows a crosssectional microscope image of a strip waveguide clad with $9.3 \mu \mathrm{m}$ of planarised PECVD silica. The strip waveguides have slightly trapezoidal cross-sections, with a top corner angle of $96^{\circ} \pm 1.4^{\circ}$ which is not expected to significantly affect their operation [32].

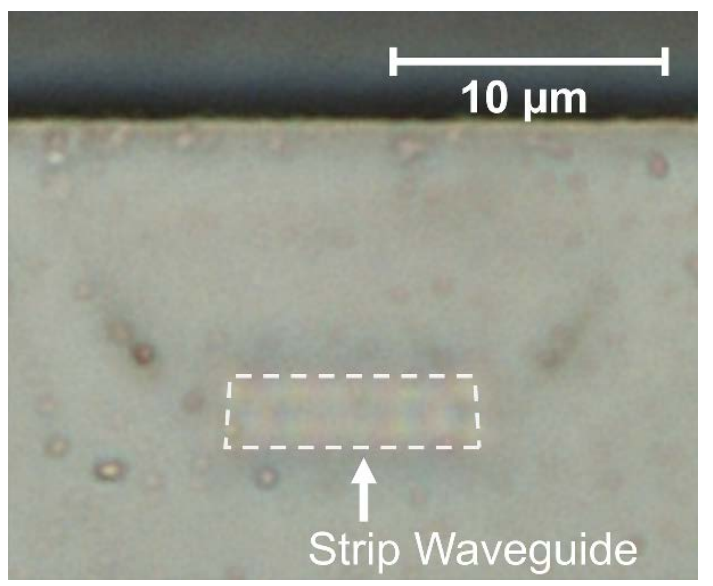

Figure 4. Cross-sectional microscope image of the waveguide end facet after planarisation.

To produce deep microfluidic channels, high etch selectivity between the doped and undoped silica and the chromium hard mask is required. The etch rate and selectivity of bulk silica/chromium of 44:1 is comparable to other silica/hard mask systems reported in the literature [33,34] and enabled etching of microfluidic channels up to $35 \mu \mathrm{m}$ in depth. High aspect ratio, rectangular cross-section microfluidic channels suitable for inertial focussing were fabricated. Figure 5 shows a cross-sectional SEM image of the highest aspect ratio microfluidic channel produced with a height/width ratio of 2.1:1 and side wall verticality of $91^{\circ} \pm 4^{\circ}$. Some trenching at the bottom corners due to bombardment 
of the substrate at high ion energy was observed and was shown to be reduced with lower RF bias power at the cost of slower etch rate. The relatively small trench features $\left(\sim 1 \times 3 \mu \mathrm{m}^{2}\right)$ did not significantly affect the location of the bead equilibrium positions as it was found that all beads were still observed to flow through the illumination beam.

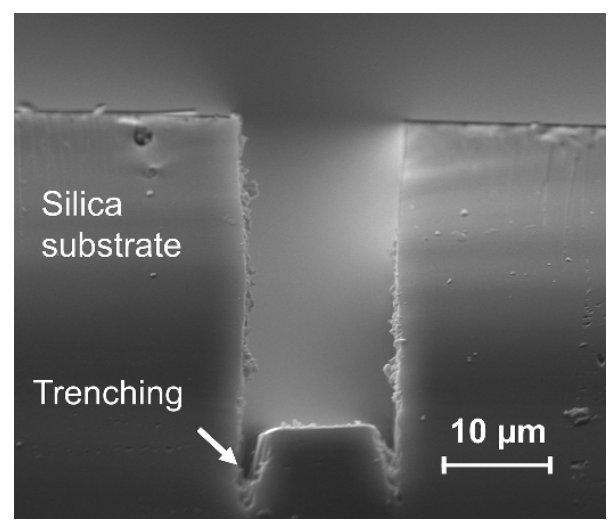

Figure 5. Cross-sectional SEM image of a microfluidic channel $13 \mu \mathrm{m}$ wide by $28 \mu \mathrm{m}$ deep etched into a silica substrate.

Figure 6 shows an SEM image of an angled view of the side wall of a microfluidic channel used for process validation, etched through the three optical layers without planarisation. The end facet of a $1.8 \mu \mathrm{m}$ wide monomode strip waveguide is clearly defined. The $2.4 \mu \mathrm{m}$ high ridge on the floor of the microfluidic channel is due to the original cladding surface being ridged before etching. These 'micro-weir' structures can cause redirection of flow [35] and so these features were prevented by planarising the chips before etching of the microfluidic channels in the final devices.

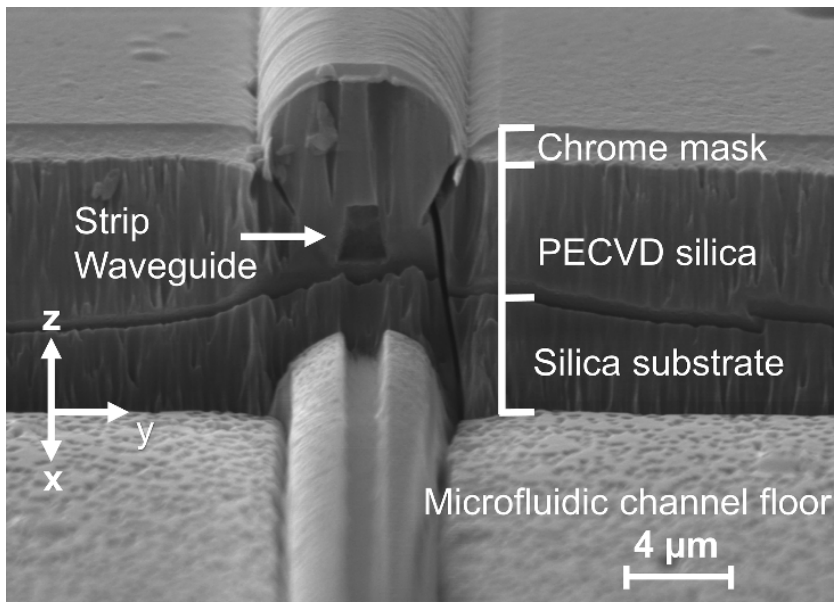

Figure 6. Tilted SEM image of the side wall of an etched $8 \mu \mathrm{m}$ deep microfluidic channel with a $1.8 \mu \mathrm{m}$ strip waveguide without surface planarisation.

This optimised fabrication procedure was used to produce devices for optical characterisation and for fluorescence and transmission measurements with flowing beads.

\subsection{Optical characterisation}

Waveguides of width $2 \mu \mathrm{m}$ were confirmed to be single-mode at a wavelength of $532 \mathrm{~nm}$ while wider waveguides were multimode. The losses incurred for waveguides crossing a $20 \mu \mathrm{m}$ wide channel filled with an aqueous solution of $0.2 \% \mathrm{v} / \mathrm{v}$ Tween 20 surfactant in the absence of beads were $3.6 \mathrm{~dB}$ and $3.5 \mathrm{~dB}$ for $2.0 \mu \mathrm{m}$ and $9.2 \mu \mathrm{m}$ wide waveguides respectively. This loss is predominantly due to diffraction of the beam across the microfluidic channel which is predicted by a Gaussian approximation to be $2.2 \mathrm{~dB}$ for a beam with an initial half-width of $1.25 \mu \mathrm{m}$ which is equivalent to the beam produced by the $2 \mu \mathrm{m}$ wide waveguide [36]. The contribution to loss from 
Fresnel reflection is minimal at $0.2 \%$ per interface and absorption in the channel is negligible. The excess loss of approximately $1 \mathrm{~dB}$ is believed to be due to scattering at the etched waveguide facets. These losses are in the same order as those reported in the literature [17,37] and enable straightforward transmission-based detection of flowing beads. Figure 7 shows example images of beams crossing the channel, as revealed by the fluorescent dye solution, for a $2 \mu \mathrm{m}$ and a $9.2 \mu \mathrm{m}$ wide waveguide. Intensity profiles across the beams at the left and right equilibrium positions 6.8 $\mu \mathrm{m}$ from each sidewall, as discussed in Section 3.3, are also shown. Figure 7(a) shows that the narrowest beam was at the output of the $2 \mu \mathrm{m}$ wide waveguide, and the beam widths (full width at $1 / \mathrm{e}^{2}$ maximum intensity) were $4.3 \mu \mathrm{m}$ and $5.6 \mu \mathrm{m}$ for the right and left sides, respectively. The widest beam produced, shown in Figure 7(b), was from a (multimode) $9.2 \mu \mathrm{m}$ wide waveguide which yielded a width of $9.0 \mu \mathrm{m}$ at the right equilibrium position and $9.9 \mu \mathrm{m}$ at the left equilibrium position. The wider waveguide exhibits a slightly lower loss due to this lower divergence. As all the waveguides have a thickness of $2 \mu \mathrm{m}$ it is expected that diffraction in the vertical plane (XZ) for all waveguide widths is approximated by the horizontal diffraction (XY) observed for the $2 \mu \mathrm{m}$ width waveguide. Diffraction in the vertical plane is therefore a major contributor to loss across the channel for all waveguide widths. Figure $7(\mathrm{~b})$ shows that the $9.2 \mu \mathrm{m}$ wide waveguide launched a dual-lobed intensity distribution across the channel, which is due to the multimode nature of the waveguide. The width of the beam determines the illumination time and intensity as a bead passes through it. In this work the $9.2 \mu \mathrm{m}$ wide waveguide was used to illuminate flowing beads as the wider beam allows a greater total illumination energy without saturation of the CCD, resulting in a greater total fluorescence energy measured, and therefore higher signal to noise ratio than the narrower $2 \mu \mathrm{m}$ waveguide. The non-uniformity of the illumination from the $9.2 \mu \mathrm{m}$ wide waveguide is not expected to affect bead fluorescence measurements significantly as all beads pass through the full width of the beam and the signal is the received power integrated over the time the bead is in the beam. 

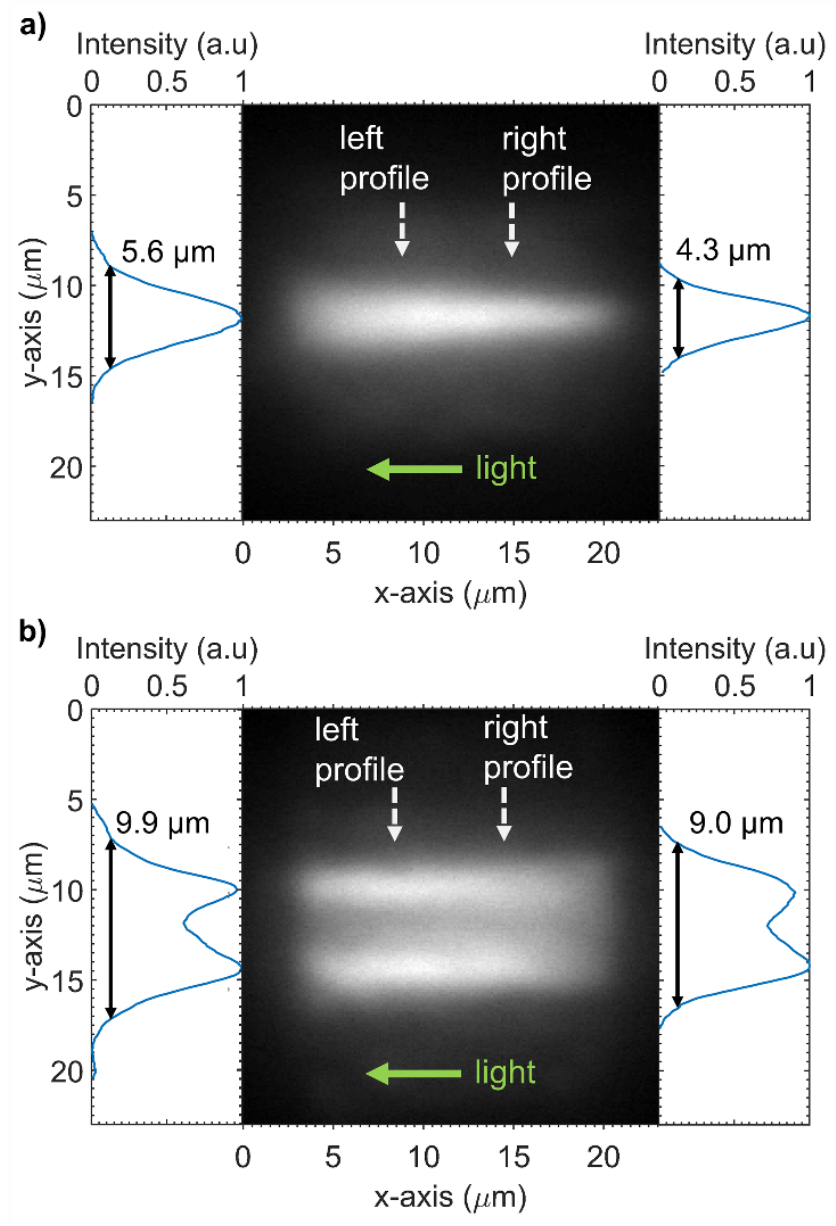

Figure 7. Fluorescence microscope images of the beam crossing the microfluidic channel with accompanying graphs showing the intensity distributions parallel to the $Y$ axis for the right and left inertial focussing positions for (a) a $2 \mu \mathrm{m}$ wide single mode waveguide and (b) a $9.2 \mu \mathrm{m}$ wide multimode waveguide.

\subsection{Inertial focussing characterisation}

The extent to which inertial focussing achieved confinement of the bead flow streams was assessed. Well-confined flow streams are expected to reduce signal variation by minimising variation in the excitation power falling on the bead and also to maximise the proportion of beads flowing through the beam. The $9.2 \mu \mathrm{m}$ wide waveguide was used to excite bead fluorescence as it was shown to produce approximately twice the fluorescence energy as excitation with the $2 \mu \mathrm{m}$ wide waveguide as the bead illumination time was approximately doubled. This difference in fluorescence signal was due to the use of a single CCD image acquisition to integrate the entire fluorescence power produce by a bead over time. Figure 8 shows fluorescence image with the averaged fluorescence from 522 individual fluorescent beads which flowed through the beam over $240 \mathrm{~s}$, driven at a flow rate of 0.2 $\mu \mathrm{L} / \mathrm{s}$. The image shows that the beads are tightly confined into left and right equilibrium positions centred $6.8 \mu \mathrm{m} \pm 0.8 \mu \mathrm{m}$ from the microfluidic channel walls which indicates that inertial focussing is well developed parallel to the short face of the channel ( $X$ axis). The dual lobed $Y$ intensity distribution observed in Figure 7 is again revealed in Figure 8 as the beads produce fluorescence directly proportional to the excitation intensity as they pass through the beam. The intensity distribution is slightly different to that observed in Figure $7(b)$. This is due to a small change in the position of the input fibre between experiments causing altered modal excitation. The ratio of the number of beads to pass on the left vs right equilibrium positions varied between experiments from $1: 1$ to as high as 1:260. The cause of a bias was attributed to asymmetric feeding of beads into the channel due to the variable positioning of the input tubing over the reservoir. At a flow rate of 0.2 
$\mu \mathrm{L} / \mathrm{s}$, the bead count rate was 124 beads/minute demonstrating that $\sim 100 \%$ of the beads injected into the chip flowed through the beam. If flowing beads were randomly distributed in the $Z$ dimension then only $25 \%$ of beads would be expected to flow through the beam based on the predicted dimensions of the beam in the XZ plane. This shows that inertial focussing parallel to the long face of the channel is confining all beads to flow through the beam $[18,38]$. Reducing the flow rate to $0.1 \mu \mathrm{L} / \mathrm{s}$ reduces the proportion of beads flowing through the beam to $\sim 75 \%$, showing that confinement in a fluid stream reduces at the lower flow rate.

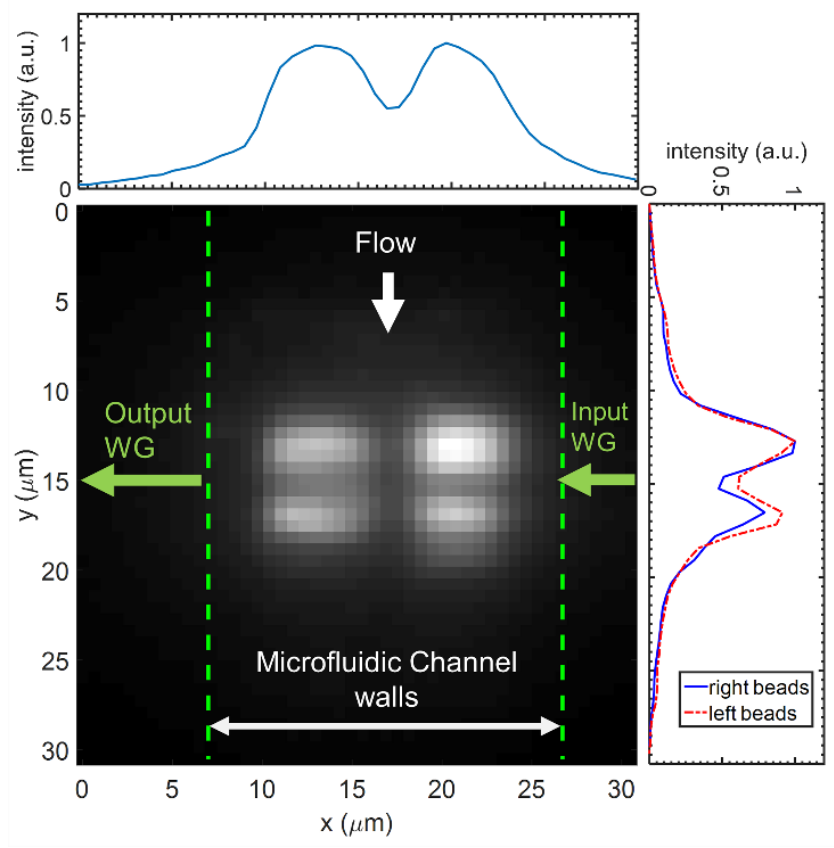

Figure 8. Fluorescence microscope image showing the averaged fluorescence from 522 beads which have flowed through the beam. Intensity in the $Y$ direction is proportional to the excitation beam intensity.

\subsection{Measurement of bead fluorescence}

Quantitative analysis of the fluorescence signal was conducted using fluorescence collected from the right equilibrium position as more beads passed on this side during the data acquisition period. Figure 9(a) shows the fluorescence energy collected by the CCD camera over an 80 s period for beads pumped at a flow rate of $0.2 \mu \mathrm{L} / \mathrm{s}$ and Figure 9(b) shows a detailed sample of $1 \mathrm{~s}$ duration. The standard deviation of the baseline signal when only buffer is flowing, representative of system noise, is $0.7 \mathrm{fJ}$. Figure 9(a) shows that there are some high peaks, and these are attributed to multiple beads passing through the imaging region during the exposure time for a single frame. These signals were not excluded from the data analysis but only represented $1.7 \%$ of the total number of signal peaks so had minimal effect on the signal quantification. Coincidences could be reduced to below $0.5 \%$ by using a bead concentration of $<4$ beads $/ \mu \mathrm{L}$ or by using a faster camera which could acquire video at $>198 \mathrm{fps}$. The CCD camera was required in this study to enable detailed measurements of illumination beam distribution and full characterisation of the inertial focussing, in future the use of a low-cost photodetector with a receiver bandwidth in the $\mathrm{MHz}$ range would allow discrimination between bead signals with a short separation time eliminating coincident signals. Figure 9(c) presents a histogram of the fluorescence energies of all the beads to pass through the equilibrium position during the $240 \mathrm{~s}$ acquisition period. The distribution is represented by a median fluorescence energy of $658 \mathrm{fJ}$. At the flow rate used, of $0.2 \mu \mathrm{l} / \mathrm{s}$, the coefficient of variation was $26 \%$ which is slightly higher than a inertial focussing microflow cytometer with external optics [39]. It is expected that higher flow rates would lead to further vertical confinement and reduce the fluorescence signal CV further as inertial lift forces increase with flow velocity causing faster 
migration of beads to equilibrium positions. Analysis at higher flow rates were prevented however as the microfluidic lid started to delaminate at rates above $0.2 \mu \mathrm{L} / \mathrm{s}$. The development of on chip fluorescence collection with either a robustly attached detector or an angled collection waveguide would also be expected to reduce the signal variation caused from vibration of the microscope fluorescence collection system used for these experiments. The device throughput of 2 beads $/ \mathrm{s}$ is low for a bead fluorometer but was necessary to prevent coincident signals whilst characterising the system using a CCD camera. While a CCD camera does not allow high bead concentrations, it does allow collection of information on spatial distributions of beads, important for detailed study of device operation. Following these detailed device studies, throughput could be greatly increased by increasing the bead concentration and using faster detectors. The self-ordering capability of inertial focussing has allowed analysis rates up to $10^{5} / \mathrm{s}$ in a single channel to be demonstrated [24].

To study the use of the device for bead-based immunoassays, where fluorophores are attached to the surface of the beads rather than being distributed through the bead volume, the fluorescence from immunoassay beads (Human TNF $\alpha$ magnetic Luminex performance assay: LUHM210, R\&D systems, USA) incubated with a $154 \mathrm{pg} / \mathrm{mL}$ sample of human TNF $\alpha$ was measured, immediately followed by measurement of the fluorescence from calibration beads. Preliminary results showed that immunoassay beads yielded a strong signal with a signal to noise ratio of $\sim 70$, indicating that the microflow cytometer was appropriate for application to bead-based immunoassays. However there was additional fluorescence signal variation ( $\mathrm{CV}=74 \%$ ) compared to the calibration beads $(\mathrm{CV}=29 \%)$, which was attributed to a non-optimised preliminary immunoassay protocol. Future work will aim to optimise the immunoassay protocol and fully calibrate the device for detection of human TNF $\alpha$, an analyte of broad interest to the inflammation research community. 

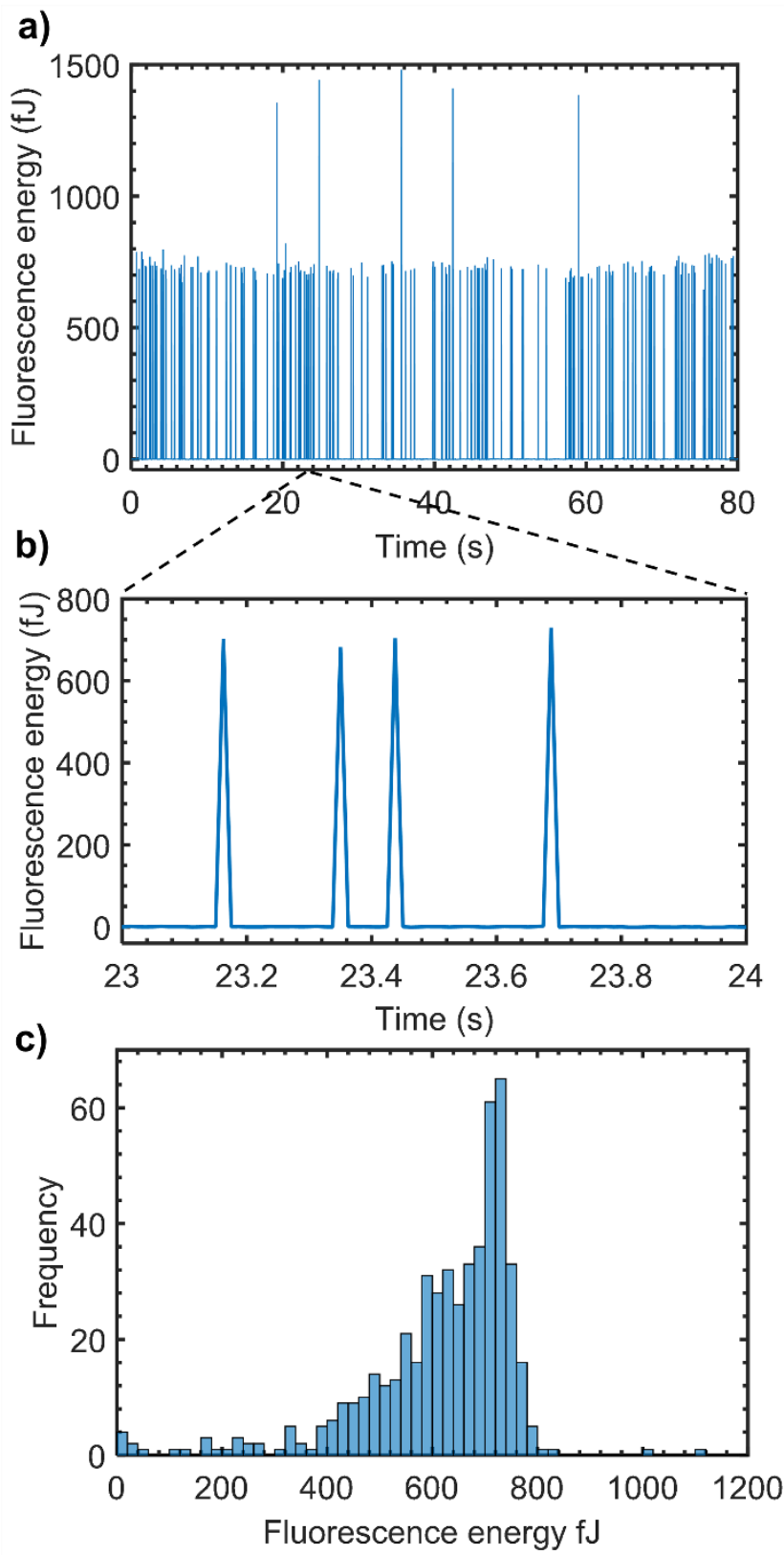

Figure 9. (a) Fluorescence energy collected by the CCD camera for beads flowing at $0.2 \mu \mathrm{L} / \mathrm{s}$ through the beam from a 9.2 $\mu \mathrm{m}$ wide waveguide. (b) Zoomed in part of (a). (c) Histogram of bead fluorescence energies from beads passing on the right equilibrium position.

\subsection{Measurement of bead transmission signal}

Bead transmission signals were collected to evaluate the change in transmitted power for nominally identical beads. The transmission signal for 12 inertially focused beads driven at a flow rate of 0.2 $\mu \mathrm{L} / \mathrm{s}$ were compared to 3 randomly positioned beads driven at a flow rate of $0.05 \mu \mathrm{L} / \mathrm{s}$. As all beads were of the same diameter, the amplitude of the transmission decrease relates to the $Z$ displacement of each bead from the optical axis and provides a measure of the confinement of the bead flow in the $Z$ dimension due to inertial focussing. The standard deviation of the baseline transmission measured when only buffer was flowing, representative of system noise, was $1.2 \%$. Figure 10(a) and (b) show the change in transmittance across the fluidic channel measured from the output waveguide as beads flow through the beam, normalised to the transmittance with buffer solution alone flowing in the channel, for flow rates of $0.2 \mu \mathrm{L} / \mathrm{s}$ and $0.05 \mu \mathrm{L} / \mathrm{s}$, respectively. In Figure 
10(a), in the case of the higher flow rate, beads 1 and 2 show decreases in transmission of $71 \%$ and $59 \%$ respectively indicating that a large proportion of the optical power leaving the input waveguide is absorbed or deflected from coupling to the output waveguide mode by the bead. Bead 1 also shows two transmission minima, which may be due to the bead passing through the two beam intensity maxima observed in Figure 7 and the two peak fluorescence positions shown in Figure 8. Beads 3 and 4 interrupt the transmission less significantly, which is due to imperfect inertial focussing causing the beads to deviate from the centre of the beam. The beam interruption is also of shorter duration because the full width of the bead is not passing through the centre of the beam.

a) $\quad$ Flow rate $0.2 \mu \mathrm{L} / \mathrm{s}$

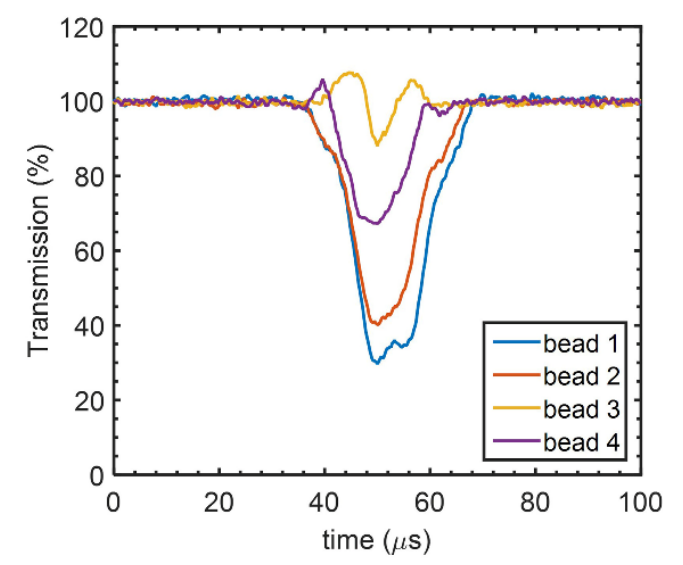

b) $\quad$ Flow rate $0.05 \mu \mathrm{L} / \mathrm{s}$

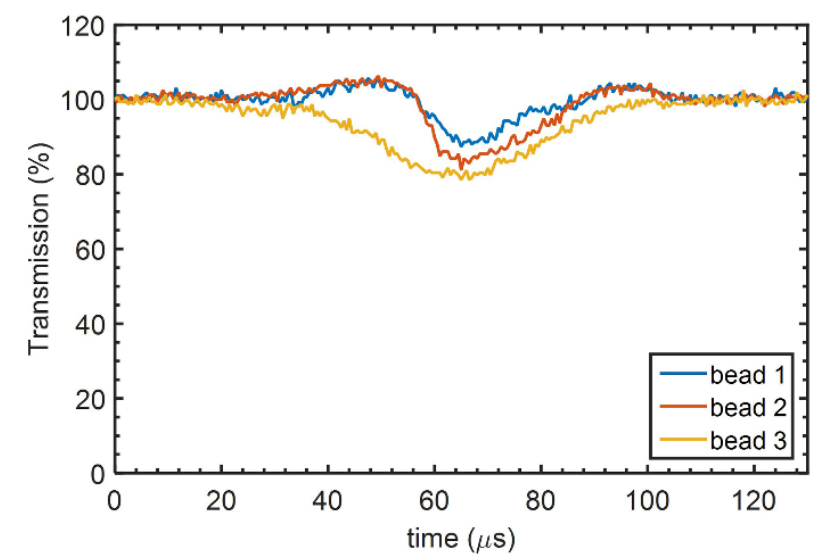

Figure 10. Modulation in microfluidic channel transmission as beads flow through the beam for two flow rates (a) 0.2 $\mu \mathrm{L} / \mathrm{s}$ and (b) $0.05 \mu \mathrm{L} / \mathrm{s}$, multiple bead signals are overlaid for comparison

In Figure 10(b), all beads show a smaller decrease in transmission as the flow rate is too slow to produce inertial focussing which greatly reduces the chance of a bead passing through the beam in line with the optical axis and causing maximum attenuation. The duration of the beam interruption is also extended, as expected at the lower flow rate. An increase in transmitted power before and after the transmission minimum is observed for bead 3 in Figure 10(a) and at the lower flow rate for beads 1 and 2 in Figure 10(b). This increase in transmission is due to a bead focussing or scattering increased power into the output waveguide when it passes above or below the waveguide axis.

The transmission measurements observed provide complementary information to fluorescence measurements, with high signal strength, offering the potential for particle sizing, data 
synchronisation and ultimately the potential for forward and side scattering measurements with angled and offset collection waveguide designs.

\section{CONCLUSIONS}

A glass-based microflow cytometry platform which combines integrated optics with inertial focussing has been demonstrated and applied to performing a bead-based immunoassay. The system integrates a wave guiding plane with fluidics suitable for inertial focussing, fabricated using microelectronics techniques suitable for mass-production. Monolithic fabrication using chemically and mechanically robust materials offers the advantages of precise and stable alignment with long lifetime and with reduced assembly costs compared to traditional flow cytometers. Waveguide endfacets embedded in the microfluidic channel walls launch excitation beams across the channels, which are collected by waveguides embedded in the opposite wall. Beam widths of order 4-10 $\mu \mathrm{m}$ have been achieved with cross-channel transmission losses of $\sim 3.5 \mathrm{~dB}$, suitable for transmissionbased detection of flowing particles. The addition of further collection waveguides offers the potential for forward-scatter and side-scatter measurements. Observation of fluorescent calibration beads flowing at rates up to the chip pressure limit, using a CCD camera, has shown that there is confinement of bead flow streams due to inertial focussing. Full confinement was shown to occur parallel to the short face of the channel, creating left and right equilibrium positions. Beads were also well confined parallel to the long face of the channel so that all beads flowed through the excitation beam positioned halfway up the height of the channel. The measured fluorescence signal CV for calibration beads of $26 \%$ was slightly higher than another inertial focussing based cytometer with external optics [39]. The use of higher flow rates to further confine the flow streams and an on chip fluorescence collection system to minimise the effect of vibration are expected to further reduce the fluorescence signal variation. Using a higher flow rate and bead concentration will also increase device throughput to a similar rate used in commercial bead fluorimeters. Preliminary measurements of immunoassay beads incubated with the cytokine TNF $\alpha$ show promising results, although further development of the immunoassay protocol is required to perform a full quantitative study. Finally beads were detected by measuring the change in transmitted power between the input and output waveguides and the possible future applications of this method were discussed.

This flexible microflow cytometry platform can be adapted to a variety of applications including counting and analysis of cells and extracellular vesicles. The beam width can be tailored to the particle of interest by varying the waveguide dimensions to match the particle diameter in order to prevent coincident signals. The present channel geometry can be used for inertial focussing of particles in a size range from 3-10 $\mu \mathrm{m}$ with adjustment of the flow rate and larger channel widths can be used for larger particles. This device is primarily intended as a replacement for a bench top cytometer used in a research or hospital laboratory, allowing the immunoassay reaction to be performed using standard procedures. The compact and robust integration of the principal optical and fluidic components offers potential for future deployment as a point-of-care device, as alignment and vibration issues will be reduced, but further developments such as permanent attachment of fibre pigtails, simplified fluorescence detection and a portable immunoassay procedure will be required.

Data used in this publication can be accessed at http://dx.doi.org/10.5258/SOTON/391390.

\section{ACKNOWLEDGEMENTS}

The authors would like to thank the European Research Council for funding this work under the European Union's Seventh Framework Programme (FP7/2007-2013) ERC grant agreement no. 
291216 "Wideband Integrated Photonics for Accessible Biomedical Diagnostics". The authors also thank Mike Perry for his advice in the cleanroom, Robert Standish for machining of cover slips and Dr Jonathan West for microfluidics advice.

\section{REFERENCES}

[1] Ateya D A, Erickson J S, Howell P B, Hilliard L R, Golden J P and Ligler F S 2008 The good, the bad, and the tiny: A review of microflow cytometry Anal. Bioanal. Chem. 391 1485-98

[2] Henriquez R R, Ito T, Sun L and Crooks R M 2004 The resurgence of Coulter counting for analyzing nanoscale objects Analyst 129 478-82

[3] Cheung K, Gawad S and Renaud P 2005 Impedance Spectroscopy Flow Cytometry : On-Chip Label-Free Cell Differentiation Cytom. part A 132 124-32

[4] Spencer D, Elliott G and Morgan H 2014 A sheath-less combined optical and impedance micro-cytometer Lab Chip 14 3064-73

[5] Monat C, Domachuk P and Eggleton B J 2007 Integrated optofluidics: A new river of light Nat. Photonics 1 106-14

[6] Shapiro H M and Hercher M 1986 Flow cytometers using optical waveguides in place of lenses for specimen illumination and light collection. Cytometry $7221-3$

[7] Nguyen N-T, Lassemono S and Chollet F A 2006 Optical detection for droplet size control in microfluidic droplet-based analysis systems Sensors Actuators B Chem. 117 431-6

[8] Golden J P, Kim J S, Erickson J S, Hilliard L R, Howell P B, Anderson G P, Nasir M and Ligler F S 2009 Multi-wavelength microflow cytometer using groove-generated sheath flow. Lab Chip 9 1942-50

[9] Butement J T, Hunt H C, Rowe D J, Karabchevsky A, Hua P, Muragan G S, Clark O, Holmes C, Carpenter L G, Gates J C, Smith P G R, Chad J E and Wilkinson J S 2014 A microflow cytometer for microsphere-based immunoassays using integrated optics and inertial particle focussing Biosensors '14

[10] Watts B R, Zhang Z, Xu C Q, Cao X and Lin M 2014 Scattering detection using a photonicmicrofluidic integrated device with on-chip collection capabilities Electrophoresis 35 271-81

[11] Watts B R, Zhang Z, Xu C Q, Cao X and Lin M 2012 A photonic-microfluidic integrated device for reliable fluorescence detection and counting Electrophoresis 33 3236-44

[12] Kotz K T, Petrofsky A C, Haghgooie R, Granier R, Toner M and Tompkins R G 2013 Inertial focusing cytometer with integrated optics for particle characterization Technology 1 27-36

[13] Hunt H C and Wilkinson J S 2012 Kinoform microlenses for focusing into microfluidic channels. Opt. Express $209442-57$

[14] Hunt H C and Wilkinson J S 2011 Multimode interference devices for focusing in microfluidic channels. Opt. Lett. 36 3067-9

[15] Suzuki K, Hida Y, Shibata T, Inoue Y, Takahashi H and Okamoto K 2006 Silica-based arrayedwaveguide gratings for the visible wavelength range NTT Tech. Rev. 4 48-51

[16] Friis P, Hoppe K, Leistiko O, Mogensen K B, Hübner J and Kutter J P 2001 Monolithic integration of microfluidic channels and optical waveguides in silica on silicon. Appl. Opt. $\mathbf{4 0}$ 6246-51 
[17] Ou H 2004 Trenches for Building Blocks of Advanced Planar Components IEEE Photonics Technol. Lett. 16 1334-6

[18] Amini H, Lee W and Di Carlo D 2014 Inertial microfluidic physics. Lab Chip 14 2739-61

[19] Zhou J and Papautsky I 2013 Fundamentals of inertial focusing in microchannels. Lab Chip 13 1121-32

[20] Di Carlo D, Irimia D, Tompkins R G and Toner M 2007 Continuous inertial focusing, ordering, and separation of particles in microchannels. Proc. Natl. Acad. Sci. U. S. A. 104 18892-7

[21] Howell P B, Golden J P, Hilliard L R, Erickson J S, Mott D R and Ligler F S 2008 Two simple and rugged designs for creating microfluidic sheath flow. Lab Chip 8 1097-103

[22] Holmes D, She J K, Roach P L and Morgan H 2007 Bead-based immunoassays using a microchip flow cytometer. Lab Chip 7 1048-56

[23] Edd J F, Di Carlo D, Humphry K J, Köster S, Irimia D, Weitz D a and Toner M 2008 Controlled encapsulation of single-cells into monodisperse picolitre drops. Lab Chip 8 1262-4

[24] Hur S C, Tse H T K and Di Carlo D 2010 Sheathless inertial cell ordering for extreme throughput flow cytometry. Lab Chip $10274-80$

[25] Oakey J, Applegate R W, Arellano E, Di Carlo D, Graves S W and Toner M 2010 Particle focusing in staged inertial microfluidic devices for flow cytometry. Anal. Chem. 82 3862-7

[26] Kettman J R, Davies T, Chandler D, Oliver K G and Fulton R J 1998 Classification and properties of 64 multiplexed microsphere sets Cytometry 33 234-43

[27] Welsh J, Holloway J and Englyst N 2014 Internal Medicine : Open Access Microvesicles as Biomarkers in Diabetes, Obesity and Non-Alcoholic Fatty Liver Disease : Current Knowledge and Future Directions

[28] Matzdorff a C, Kühnel G, Kemkes-Matthes B and Pralle H 1998 Quantitative assessment of platelets, platelet microparticles, and platelet aggregates with flow cytometry. J. Lab. Clin. Med. 131 507-17

[29] Messow F, Welch C, Eifert A, Ang W C, Hoe N S, Kusserow T and Hillmer H 2014 Deep single step vertical ICP-RIE etching of ion beam sputter deposited SiO2/Si multilayer stacks Microelectron. Eng. 113 70-3

[30] Hamamatsu Photonics 2015 ORCA R2 Digital CCD Camera

[31] Kim, Y. T., Cho, S. M., Seo, Y. G., Yoon, H. D. Im, Y. M., Yoon D H 2002 Influence of hydrogen on $\mathrm{SiO} 2$ thick film deposited by PECVD and FHD for silica optical waveguide Cryst. Res. Technol. 37 1257-63

[32] Hunt H C 2010 Integrated Microlenses and Multimode Interference Devices for Microflow Cytometers (University of Southampton)

[33] Kolari K, Saarela V and Franssila S 2008 Deep plasma etching of glass for fluidic devices with different mask materials J. Micromechanics Microengineering 1864010

[34] Bliznetsov V, Lin H M, Zhang Y J and Johnson D 2015 Deep SiO 2 etching with Al and AIN masks for MEMS devices J. Micromechanics Microengineering 2587002

[35] Lee H C, Hou H H, Yang R J, Lin C H and Fu L M 2011 Microflow cytometer incorporating sequential micro-weir structure for three-dimensional focusing Microfluid. Nanofluidics 11 469-78 
[36] Ajoy G and K. T 1998 Introduction to fibre optics (Cambridge university press)

[37] Choi D, Lee J, Kim D and Jung S 2003 Modification of sidewall roughness in silica deep etching and their influences on coupling loss in hybrid integration Proc. SPIE 4944 337-45

[38] Choi Y-S, Seo K-W and Lee S-J 2011 Lateral and cross-lateral focusing of spherical particles in a square microchannel. Lab Chip 11 460-5

[39] Bhagat A A S, Kuntaegowdanahalli S S, Kaval N, Seliskar C J and Papautsky I 2010 Inertial microfluidics for sheath-less high-throughput flow cytometry Biomed. Microdevices 12 18795 